UMR 5824

93, chemin des Mouilles 69130 Ecully - France

Maison de I'Université, Bâtiment 10, rue Trefilerie 42023 Saint-Etienne cedex $02 \cdot$ France

http://wwwgatecnisfr gate@gate.cnrs.fr

WP 2103 - January 2021

\title{
The T-periodic choice with limited loyalty
}

Muhammad Mahajne

\begin{abstract}
:
We study a decision-maker who selects, in every fixed period of time, T times from every collection of feasible alternatives, where $\mathrm{T}$ is pre-determined by him. We axiomatize the class of $\mathrm{T}$-choice functions according to which the decision-maker has fixed preference relation along the same period but has limited loyalty. The decision-maker selects, deterministically, in a way such that the higher an alternative is ranked by his preference relation the higher it is selected in his $\mathrm{T}$ choices.
\end{abstract}

\section{Keywords:}

Choice function, Axiomatization

JEL codes:

D01

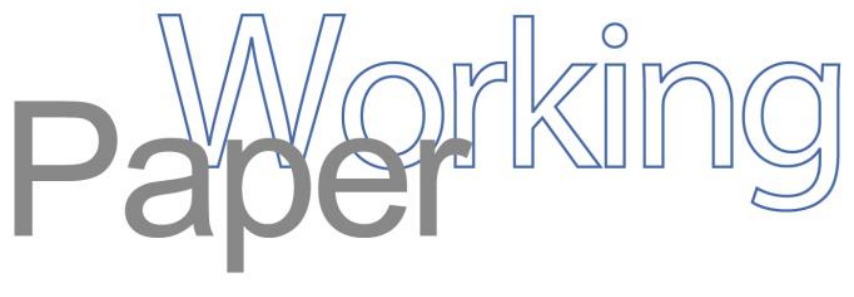




\title{
The T-periodic choice with limited loyalty*
}

\author{
Muhammad Mahajne ${ }^{\dagger}$
}

December 29, 2020

\begin{abstract}
We study a decision-maker who selects, in every fixed period of time, $\mathrm{T}$ times from every collection of feasible alternatives, where $\mathrm{T}$ is pre-determined by him. We axiomatize the class of T-choice functions according to which the decision-maker has fixed preference relation along the same period but has limited loyalty. The decision-maker selects, deterministically, in a way such that the higher an alternative is ranked by his preference relation the higher it is selected in his $\mathrm{T}$ choices.
\end{abstract}

Keywords Choice function. Axiomatization.

JEL Classification D01.

\section{Introduction}

We study a decision-maker who selects, in every fixed period of time, $\mathrm{T}$ times from every collection of feasible alternatives, where $\mathrm{T}$ is pre-determined by him. We start with the following example:

Example 1. A consumer of hair shampoo, has fixed preferences regarding the shampoo brands that are available at the point of sale where he buys. This consumer has a fixed buying pattern, according to which, with out of every four shampoo purchases, in the first three of them he chooses his most favourite brand, and in the fourth he chooses the second

*The results in this preliminary version are not for citation, some definitions and results may have to be improved or corrected. The author acknowledges financial support from Université de Lyon (project INDEPTH Scientific Breakthrough Program of IDEX Lyon) within the program Investissement d'Avenir (ANR-16-IDEX-0005).

${ }^{\dagger}$ Univ Lyon, UJM Saint-Etienne, CNRS, GATE L-SE UMR 5824, F-42023 Saint-Etienne, France. Email: mahajnah@gate.cnrs.fr 
brand in his preferences. The consumer may provide some explanations for his consumer behaviour, as: boring to use the same brand all the time, good for hair health to change the shampoo occasionally.

This example may reflect realistic buying patterns, in some consumption categories, of a consumer whose preference is stable, but whose loyalty to the preferred brand is limited.

As mentioned before, in this paper we study a decision-maker who faces, in every period of time, collections of alternatives, from an universal set of alternatives, and has to choose, deterministically, $\mathrm{T}$ times (T-variety) from every collection $A$. A T-variety is a vector of non-negative integers, whose sum is $\mathrm{T}$, which represents the number of times each alternative is selected during the period. The decision-maker is characterized by a preference relation which does not change along the same period, and by a choice function that represents his distribution of T-choices, from each collection, throughout the period. Roughly, we say that a T-choice function $\mathrm{D}$ is limited-loyalty rationalizable, if there is an ordering such that the T-variety selected by it from any collection, is compatible with this ordering (i.e., the higher an alternative is ranked by the ordering the higher its frequency in the T-variety).

Kalai, Rubinstein, and Spiegler (2002) and Rubinstein and Salant (2012) studied situations in which a decision-maker presents several preference relations or multiple rationales, each of which is used or appropriate for some circumstances or choice situations. Unlike them, we examine situations in which, the decision maker has one fixed preference relation which does not change under different circumstances, but because of his limited loyalty to his most favourite alternative, he may choose in any fixed period of time, deterministically, also other alternatives with some reasonable frequency.

We presents an axiomatization of the class of limited-loyalty rationalizable T-choice functions by means of two axioms.

\section{Definitions}

Let $X=\left\{x_{1}, \ldots, x_{K}\right\}$ be a finite set of alternatives and let $\mathcal{X}$ be the set of non-empty subsets of $X$. Elements of $\mathcal{X}$ are called menus. For any menu $A \in \mathcal{X}, n_{A}$ denotes the cardinality of $A$. Let $\mathrm{T}$ be a positive integer (pre-determined by the consumer). A Tperiodic choice function (henceforth T-choice function) is a function $D$ that assigns to every menu $A \in \mathcal{X}$, a T-variety $v(A)$, which is vector of $n_{A}$ non-negative integers, such

that their sum is $\mathrm{T}$, where $v_{j}(A)$ is the number of times in which $x_{j}$ appears in the 
T-variety $v(A)$. For an individual preference relation (linear order) $\succ=\left(b_{1}, \ldots, b_{K}\right)$ on $X$, and given a set of alternatives $A \in \mathcal{X}$, the $n_{A}$-vector $v(A)$ of sum $\mathrm{T}$, is compatible with $\succ$ (or $\succ$-compatible) if $v_{i}(A) \geq v_{j}(A)$ for all $i, j$ such that $b_{i}, b_{j} \in A$ and $b_{i} \succ b_{j}$.

Definition 1. A T-choice function D is limited loyalty-rationalizabe (LL-rationalizabe), if there exists a linear order $\succ=\left(b_{1}, \ldots, b_{K}\right)$ on $X$, such that $v(A)$ is $\succ$-compatible $\forall A \in \mathcal{X}$, with at least one strong inequality for every pair $b_{i}, b_{j} \in X(i<j)$ for some $A^{\prime} \in \mathcal{X}$.

For instance, the following T-choice function is LL-rationalizabe. This function selects in every period, the top ranked element from any collection in the first $(T-1)$ times and in the last choice it selects the second ranked element, according to the preference relation of the decision-maker. Also, the following T-choice function is LL-rationalizabe. This function selects each element from any collection in a frequency which reflects, inversely, its position in the preference relation of the decision-maker.

\section{Axiomatization}

T-Revealed Preference (TRP) $\forall A, A^{\prime} \in \mathcal{X}$ and $a, b \in A, A^{\prime}$, if in $D(A)$ it holds that $v_{a}(A)>v_{b}(A)$ then in $D\left(A^{\prime}\right)$ it is not that $v_{a}\left(A^{\prime}\right)<v_{b}\left(A^{\prime}\right)$.

First Element There is an alternative $x \in X$ (called first element) such that for all $A \in \mathcal{X}, x \in A \Longrightarrow v_{x}(A) \geq v_{y}(A)$ for any $y \in A$, and at least one strong inequality for every pair $x, y \in X$ (for some $A^{\prime} \in \mathcal{X}$ ).

TRP axiom requires that if two elements belong to each one of two subsets and the first element is selected from the first subset with strictly higher frequency than the second one, then it can't be that the second element is selected from the second subset with strictly higher frequency than the first one. The First Element axiom requires the existence of an alternative that is chosen with maximal frequency whenever available.

Note 1. The two axioms are independent. To see that, let $X=\left\{x_{1}, x_{2}, x_{3}\right\}$ and $T=3$. Consider first, the T-choice functions D1, according to which: $v\left(\left\{x_{1}, x_{2}, x_{3}\right\}\right)=(1,1,1)^{1}$ , $v\left(\left\{x_{1}, x_{2}\right\}\right)=(2,1), v\left(\left\{x_{2}, x_{3}\right\}\right)=(2,1)$ and $v\left(\left\{x_{1}, x_{3}\right\}\right)=(1,2)$. D1 satisfies TRP but not First Element. Consider now D2, according to which: $v\left(\left\{x_{1}, x_{2}, x_{3}\right\}\right)=(2,1,0)$, $v\left(\left\{x_{1}, x_{2}\right\}\right)=(2,1), v\left(\left\{x_{2}, x_{3}\right\}\right)=(1,2)$ and $v\left(A=\left\{x_{1}, x_{3}\right\}\right)=(3,0)$. We see that $x_{1}$ is

\footnotetext{
${ }^{1}$ We denoted that it this way for shortening, and we mean that $v_{x_{1}}\left(\left\{x_{1}, x_{2}, x_{3}\right\}\right)=1, v_{x_{2}}\left(\left\{x_{1}, x_{2}, x_{3}\right\}\right)=$ $1, v_{x_{3}}\left(\left\{x_{1}, x_{2}, x_{3}\right\}\right)=1$
} 
First Element, but D2 violates TRP (notice that $v_{x_{2}}\left(\left\{x_{1}, x_{2}, x_{3}\right\}\right)>v_{x_{3}}\left(\left\{x_{1}, x_{2}, x_{3}\right\}\right)$ but $\left.v_{x_{3}}\left(\left\{x_{2}, x_{3}\right\}\right)>v_{x_{2}}\left(\left\{x_{2}, x_{3}\right\}\right)\right)$.

The next definition is needed for the following proposition:

Definition 2. Let $D$ be a T-choice function. The D-associated binary relation $\unrhd_{R}$ on $X$ is defined by $a \unrhd_{R} b$ iff $\exists A \in \mathcal{X}$ such that in $D(A), v_{a}(A)>v_{b}(A)$ where $a \neq b \in A$.

The following proposition provides an axiomatization of the class of LL-rationalizabe T-choice functions.

Theorem 1. Let D be a T-choice function. The following three statements are equivalent:

(a) T-choice function D satisfies TRP and First Element.

(b) T-choice function $D$ is LL-rationalizabe.

(c) The associated $\unrhd_{R}$ of $D$ is linear order ${ }^{2}$.

Proof. (Part 1: $(a) \Leftrightarrow(b))$ It is easy to see that any LL-rationalizabe T-choice function $D$ satisfies the forgoing axioms. Let $D$ be a T-choice function that satisfies the axioms. We need to find a linear order $\succ$ on $X$ such that for all $A \in \mathcal{X}$, in $D(A)$ it holds that $v_{x_{i}}(A) \geq v_{x_{j}}(A)$, for all $i<j$.

The proof is by induction on the cardinality of $X$. If $|X|=1$ then, there is only one choice correspondence and it can be seen that is a LL-rationalizabe T-choice function. It is easy to see that the statement of the proposition holds for any $X$ such that $|X|=2$.

Let $K>2$ and assume that for all sets of alternatives $X^{\prime}$ with cardinality less than $K$ the statement of the proposition holds: Any T-choice function $D^{\prime}$ defined on $X^{\prime}$ that satisfies TRP and First Element is a LL-rationalizabe. Let $X=\left\{x_{1}, \ldots, x_{K}\right\}$ and assume that $D$ satisfies the axioms. By First Element, there is an element, say $x_{K} \in X$ such that for all $A \in \mathcal{X}, x_{K} \in A \Longrightarrow$ in $D(A), v_{x_{K}}(A) \geq v_{x_{j}}(A)$, for all $j<K$. Consider the set of alternatives

$$
X^{\prime}=X \backslash\left\{x_{K}\right\}
$$

Let $D^{\prime}$ be the restriction of $D$ to $X^{\prime}$. Since $D$ satisfies TRP and First Element on $X$, so does $D^{\prime}$ on $X^{\prime}$. By the induction hypothesis, there is $\succ^{\prime}$ on $X^{\prime}$ such that for all $A \in \mathcal{X}^{\prime}$, $D^{\prime}(A)$ is compatible with $\succ^{\prime}$. Let $\succ$ be the order on $X$ that is obtained by extending $\succ^{\prime}$ so that $x_{K} \succ x$ for all $x \in X^{\prime}$. Let $A \in \mathcal{X}$. We need to show that $D(A)$ is compatible with $\succ$. If $x_{K} \notin A$, then $D(A)=D^{\prime}(A)$ and we are done. If, on the other hand, $x_{K} \in A$, we can

\footnotetext{
${ }^{2} \unrhd_{R}$ is linear order if it is complete, transitive and antisymmetric
} 
write $A=A^{\prime} \cup\left\{x_{K}\right\}$, where $A^{\prime} \subset X$. By First Element, $v_{x_{K}}(A) \geq v_{x_{j}}(A)$, for all $x_{j} \in A^{\prime}$. By TRP, if $v_{x_{i}}\left(A^{\prime}\right) \geq v_{x_{j}}\left(A^{\prime}\right)$ for all $x_{i} \succ^{\prime} x_{j}$ (and at least one strong inequality for some $A^{\prime \prime} \subseteq X^{\prime}$ ), then $v_{x_{i}}(A) \geq v_{x_{j}}(A)$ for all $x_{i} \succ x_{j}$ (with at least one strong inequality for some $\left.A^{* *} \subseteq X^{\prime}\right)$. Therefore, $A$ is compatible with $\succ$.

(Part 2: $(b) \Leftrightarrow(c))$ First we show that $D$ is a LL-rationalizabe $\Rightarrow_{R}$ is linear order. Since $D$ is LL-rationalizabe, we have that $\unrhd_{R}$ complete. Let $\succ$ be the (unique) linear order that rationalizes $D$, and assume that $a \unrhd_{R} b$ and $b \unrhd_{R} c$. Then it must be that $a \succ b$ and $b \succ c$. Since $\succ$ is transitive we obtain $a \succ c$, and (since $D$ is a LL-rationalizabe) we must also have that $a \unrhd_{R} c$. Therefore, $\unrhd_{R}$ is transitive. Now, let $a \neq b$ such that $a \unrhd_{R} b$, then for any $A \in \mathcal{X}, v_{a}(A) \geq v_{b}(A)$. By axiom TRP, there is no $A \in \mathcal{X}$ such that $v_{b}(A)>v_{a}(A)$, thus it can't be that $b \unrhd_{R} a$. Therefore, $\unrhd_{R}$ is antisymmetric.

Second we show that $\unrhd_{R}$ is linear order $\Rightarrow D$ is a LL-rationalizabe. Consider $\triangleright$. For any $x_{i}, x_{j} \in X$ such that $x_{i} \triangleright x_{j}$ we have that $\forall A \in \mathcal{X}, v_{x_{i}}(A) \geq v_{x_{j}}(A)$ and at least one strong inequality for some $A \in \mathcal{X}$. We choose $\succ=\triangleright$ as a candidate to rationalize $D$. We obtain that $\succ$ is linear order, and $\forall x_{i}, x_{j} \in X$ such that $x_{i} \succ x_{j}$ we have that $\forall A \in \mathcal{X}$, $v_{x_{i}}(A) \geq v_{x_{j}}(A)$ (with at least one strong inequality for every pair $x_{i}, x_{j} \in X$ for some $\left.A^{\prime} \in \mathcal{X}\right)$. Therefore, our choice is correct.

\section{Concluding remarks}

We have axiomatized the class of T-choice functions according to which the decision-maker has fixed preference relation along the same period but has limited loyalty, and he selects in a deterministic way such that the higher an alternative is ranked by his preference relation the higher it is selected in his $\mathrm{T}$ choices.. It would be interesting to see if some families of this class can be characterized by some additional properties.

\section{References}

Kalai, G., A. Rubinstein, AND R. Spiegler (2002): "Rationalizing choice functions by multiple rationales,". Econometrica: Journal of the Econometric Society, pp. 2481-2488.

Rubinstein, A. AND Y. Salant (2012): "Eliciting welfare preferences from behavioural data sets,". Review of Economic Studies, 79, 375-387. 